\title{
Erratum: On the Noether charge and the gravity duals of quantum complexity
}

\author{
Zhong-Ying Fan ${ }^{a}$ and Minyong Guo ${ }^{b, c}$ \\ ${ }^{a}$ Center for Astrophysics, School of Physics and Electronic Engineering, \\ Guangzhou University, Guangzhou 510006, China \\ ${ }^{b}$ Department of Physics, Beijing Normal University, \\ Beijing 100875, P.R. China \\ ${ }^{c}$ Perimeter Institute for Theoretical Physics, \\ Waterloo, Ontario N2L 2Y5, Canada \\ E-mail: fanzhy@gzhu.edu.cn, guominyong@gmail.com
}

ERRATUM to: JHEP08(2018)031

KEYWORDS: AdS-CFT Correspondence, Gauge-gravity correspondence

ARXIV EPRINT: 1805.03796

In the original published version of this paper, the corresponding author was selected by mistake during the review process. Here we state that Minyong Guo is the corresponding author of this paper.

Open Access. This article is distributed under the terms of the Creative Commons Attribution License (CC-BY 4.0), which permits any use, distribution and reproduction in any medium, provided the original author(s) and source are credited. 